\title{
X-ray diffraction investigation of a three-dimensional Si/SiGe quantum dot crystal
}

\author{
Vaclav Holý \\ Department of Condensed Matter Physics, Faculty of Mathematics and Physics, Charles University, \\ Ke Karlovu 5, 12116 Prague 2, Czech Republic
}

Julian Stangl, Thomas Fromherz, Rainer T. Lechner, Eugene Wintersberger, and Günther Bauer

Institute for Semiconductor and Solid State Physics, Johannes Kepler University-Linz, AltenbergerstrASSE 69, A-4040 Linz, Austria

\author{
Christian Dais, Elisabeth Müller, and Detlev Grützmacher \\ Laboratory for Micro- and Nanotechnology, Paul Scherrer Institut, CH-5232 Villigen-PSI, Switzerland \\ (Received 23 June 2008; revised manuscript received 4 September 2008; published 26 January 2009)
}

\begin{abstract}
High-resolution $\mathrm{x}$-ray diffraction was employed to study the structural properties of a three-dimensional periodic arrangement of $\mathrm{SiGe}$ quantum dots in a Si matrix. Using extreme ultraviolet lithography at a synchrotron source a two-dimensional array of pits (period $90 \times 100 \mathrm{~nm}^{2}$ ) was defined and transferred into a (001) $\mathrm{Si}$ wafer by reactive ion etching. By molecular-beam epitaxy SiGe islands of about $30 \mathrm{~nm}$ diameter and $3 \mathrm{~nm}$ height were grown into the pits. Subsequent deposition of Si spacer layers of $10 \mathrm{~nm}$ thickness and SiGe island layers results in a three-dimensionally periodic arrangement of quantum dots, mediated by the strain fields of the buried dots. Their so far unmatched structural perfection is assessed by coplanar x-ray diffractometry using synchrotron radiation. Reciprocal-space maps around the (004) and (224) reciprocal-lattice maps were recorded and analyzed to get quantitative information on the disorder of the dot positions and to obtain the mean $\mathrm{Ge}$ content of the dots. In addition, information on the strain fields was deduced from the analysis of the diffraction data. Together with atomic force microscopy data on the island shape and size distribution, a complete structural characterization is achieved.
\end{abstract}

DOI: 10.1103/PhysRevB.79.035324

PACS number(s): 68.65.Hb, 61.05.cp, 61.46.-w

\section{INTRODUCTION}

Strain-induced ordering of self-organized grown quantum dots and islands separated by spacer layers along the growth direction has been the subject of numerous publications (for reviews, see, e.g., Refs. 1-4). For InAs islands embedded in GaAs (Refs. 5 and 6) and Ge islands in Si (Refs. 7-10) vertical ordering along the growth direction has been observed and was attributed to the elastic strain fields of buried islands. These strain fields in the spacer layer produce favorable nucleation sites on top of buried islands for the ones in the subsequent island layer. Apart from the site, the size of the buried islands, elastic anisotropy of the spacer material, the growth direction, corrugated surface morphologies, and the modulation of the chemical composition of the spacer material influence the subsequent nucleation. ${ }^{11-15}$ However, despite the fact that elastic interactions favor lateral ordering of islands as well, they turned out to be rather weak ${ }^{16}$ and only rather short-range ordering was observed in the above mentioned systems by atomic force microscopy, transmission electron microscopy, and x-ray diffraction (XRD). ${ }^{16-21}$ Apart from vertical island ordering in columnar form, in III-V, 22,23 II-VI, ${ }^{24-26}$ and IV-VI compounds ${ }^{27}$ also oblique ordering was observed, ${ }^{28}$ typically for larger spacer layer thicknesses, before for sufficiently wide spacers the ordering finally vanishes. ${ }^{29}$ For the $\mathrm{PbSe} / \mathrm{PbEuTe}$ system the formation of a three-dimensionally (3D) ordered trigonal island crystal in $\mathrm{PbSe}$ island multilayers separated by $\mathrm{PbEuTe}$ spacer layers was observed for a certain range of the spacer layer thickness for growth along an elastically soft [111] direction. ${ }^{27,29}$ This peculiar ordering was caused by the strain fields of the buried islands and the pronounced anisotropy of the elastic con- stants of these IV-VI compounds turned out to favor this particular three-dimensional periodic arrangement.

Apart from the $\mathrm{PbSe} / \mathrm{PbEuTe}$ system, the in-plane ordering achieved by self-assembly is rather far from being perfect. Consequently, some years ago a templated selfassembly of semiconductor nanostructures was introduced, ${ }^{30-37}$ combining growth on patterned substrates for the definition of an initial two-dimensionally periodic island layer with subsequent vertical ordering due to the strain fields of the buried quantum dots in a multidot layer system. ${ }^{38,39}$ So far, in particular, three-dimensionally ordered structures of InAs/GaAs (Ref. 40) and SiGe/Si (Refs. 41-44) were reported. The $\mathrm{Si} / \mathrm{Ge}$ system is of special interest since it is compatible with $\mathrm{Si}$ technology and addressable dots might be useful for electronic and optoelectronic applications ${ }^{45}$ and possibly even for realizing novel concepts in quantum computing. ${ }^{46-48}$ This requires lateral as well as vertical coupling of Ge dots, for which dense arrays of Ge quantum dots at specified locations are required.

The Ge islands in 3D arrangements fabricated and investigated in a previous study by Novák et $a l .{ }^{41}$ had rather large lateral dimensions (with diameters in excess of $100 \mathrm{~nm}$ ), thus effects of lateral confinement of carriers in the $\mathrm{Ge}$ islands were negligible. In Refs. 43 and 44, the growth of small 3D ordered $\mathrm{SiGe}$ islands was reported and their photoluminescence properties were investigated. Particular emphasis was laid on the coupling of electronic states along growth direction due to small spacer layer thicknesses.

In this work, we present a detailed structural characterization of a 3D SiGe quantum dot crystal which provides a structure suitable for vertical and lateral electronic couplings of the electron states associated with the strain fields in the $\mathrm{Si}$ 
matrix due to the presence of the $3 \mathrm{D}$ ordered Ge dots. The formation of the 3D quantum dot crystals demands the nucleation of dots at predefined sites on the substrate. Lithographic techniques are best suited for the fabrication of a two-dimensional (2D) patterned Si template with sufficient perfection in periodicity and size of the predefined nucleation sites for the dots. For this purpose, electron-beam (ebeam) lithography, ${ }^{32}$ optical interference lithography, ${ }^{33}$ and ion-beam lithography ${ }^{34,35}$ have been used to fabricate prepatterned Si substrates. Whereas the first two approaches write the pattern for the nucleation site for each individual dot sequentially, the optical interference technique allows the prepatterning of large 2D arrays in a single exposure. For e-beam and ion-beam lithographies, the determination of the location of each nucleation site is limited by the resolution of the instrument, which is typically in the range of $5 \mathrm{~nm}$, thus interdot distances may vary by a few nanometers. With conventional optical interference lithography using a wavelength $\lambda>300 \mathrm{~nm}$ it is only possible to achieve periodicities of the pattern down to about $150 \mathrm{~nm}$. Extreme ultraviolet (EUV) interference lithography combines the advantages of optical lithography with the ability to produce high-precision patterns with control in the subnanometer regime due to the small light wavelength. ${ }^{49,50}$ Using EUV, the formation of patterns with even less than $30 \mathrm{~nm}$ periodicity is feasible.

So far, 2D SiGe island arrangements and 3D arrangement in $\mathrm{Si} / \mathrm{SiGe}$ multilayers have been analyzed mainly by atomic force microscopy (AFM) and transmission electron microscopy (TEM), finding near to perfect ordering of islands. In order to achieve an even more quantitative information on the structural perfection of such a 3D SiGe quantum dot crystal fabricated by growth on a prepatterned Si substrate and subsequent deposition of a $\mathrm{Si} / \mathrm{SiGe}$ island multilayer, we employ $x$-ray diffraction reciprocal-space mapping. The aim is to study the lateral as well a vertical disorder of the dot positions in a large ensemble, exploiting the fact that XRD data are taken over much larger sampling volumes than AFM and TEM. ${ }^{20}$ Besides a detailed assessment of the positional correlations, also the Ge content in the islands and the strain distribution within and around the islands are obtained, which is important for the understanding of electronic levels and the possible formation of minibands: ${ }^{44}$ This occurs only if the size and composition distribution of the individual islands in the arrangement are small enough and the distances are regular enough. For the determination of island shape, size, and size variation, we analyze AFM images of the first and last island layers prior to capping. Due to the low capping temperature, the changes in island size and shape during overgrowth are small so that the AFM data especially on size fluctuations measured on the uncapped islands are still valid also for the buried islands.

In Sec. II the fabrication of the pit-patterned substrate and the molecular-beam epitaxy is described, followed by a presentation of the XRD and AFM data. In Sec. III the model for analysis of the X-ray data is presented taking into account the disorder and correlation functions of the dot positions. From the simulation of the diffracted intensities in Sec. IV both the strain fields and the Ge content are derived. Section $\mathrm{V}$ presents the AFM measurements; the results are discussed in Sec. VI.

\section{EXPERIMENTAL}

In order to grow 3D SiGe quantum dot crystals the initial layer of islands was deposited on a two-dimensional periodic array of pits in $\mathrm{Si}(001)$ substrates. For the prepatterning of $\mathrm{Si}(100)$ substrates the technique of interference lithography using EUV light has been employed using masks made from $\mathrm{Cr}$ gratings fabricated on $\mathrm{SiN}_{x}$ membranes. A twodimensional array of pits with lateral periodicities of 90 $\times 100 \mathrm{~nm}^{2}$ was fabricated on a field of $400 \times 600 \mu \mathrm{m}^{2}$ size. Subsequent to EUV exposure, the photoresist is developed and the pattern is transferred into the Si by reactive ion etching (RIE). A very shallow pattern of just $8-10 \mathrm{~nm}$ depth is produced. Subsequently, a $50 \mathrm{~nm}$ thick $\mathrm{Si}$ buffer layer is deposited at $300{ }^{\circ} \mathrm{C}$ by molecular-beam epitaxy (MBE). The squarelike pits etched into the Si surface are transformed into narrow inverted tiplike holes of 3-4 $\mathrm{nm}$ depth during the deposition of the Si buffer layer. Subsequent deposition of 7 ML (monolayers) of pure Ge at $450{ }^{\circ} \mathrm{C}$ leads to the formation of $\{105\}$ facetted islands in the center of the pits resulting in a uniform 2D array of Ge dots. Figure 1(a) shows an AFM image of the initial island layer in the prepatterned substrate region; in panel (b) the resulting island distribution in the unpatterned part of the substrate is shown. Panels (c) and (d) contain the distributions of island height and diameter in both regions. While on the flat substrate very broad distributions are observed in the patterned region size distributions of heights and diameters of less than $8 \%$ full width at half maximum (FWHM) are determined from AFM.

This initial 2D arrangement of Ge dots is overgrown with low-temperature Si (about $10 \mathrm{~nm}$ ). The substrate temperature is ramped from 300 to $450{ }^{\circ} \mathrm{C}$ during deposition to reduce intermixing of the $\mathrm{Ge}$ dots with the $\mathrm{Si}$ cap layer. The island multilayer is completed by a further deposition of $10 \mathrm{Ge}$ dot layers consisting of $5 \mathrm{ML}$ of Ge for each of the dot layers, separated by $10 \mathrm{~nm}$ thick Si spacer layers. A final Si layer of $10 \mathrm{~nm}$ thickness covers the last layer of dots and leads to a flat surface. Due to the filling of the pits, the dots of the first layer have a different shape and a higher aspect ratio than the $\{105\}$ facetted Ge dots grown on planar Si surfaces in the second and subsequent layers. The strain-induced vertical alignment of the dots in subsequent layers is used to grow $3 \mathrm{D}$ quantum dot crystals.

Figure 2(a) shows a TEM image of a cross sectional view of a $3 \mathrm{D}$ quantum dot crystal with their structural parameters as described above. The AFM surface scan shown in Fig. 2(c) depicts the surface after the deposition of ten island layers before the final capping with $\mathrm{Si}$. The islands on the top are $\{105\}$ facetted and have a square base. The AFM scans indicate that the lateral periodicity is well preserved and no missing or extra dots can be found in the AFM of the topmost Ge dot layer. From the AFM scans information on the sizes of the dots and their size distribution are obtained in panel (e). The height of these pyramids is $3.0 \pm 0.3 \mathrm{~nm}$ and their base length is $34 \pm 3 \mathrm{~nm}$. Apparently, the narrow size distribution and the formation of only one type of island, i.e., Ge pyramids on the prepatterned surface allows for the formation of a rather perfect 3D Ge dot crystal. For comparison, in Figs. 2(b), 2(d), and 2(f), complementary TEM micrographs and AFM surface scans are shown, respectively, for 

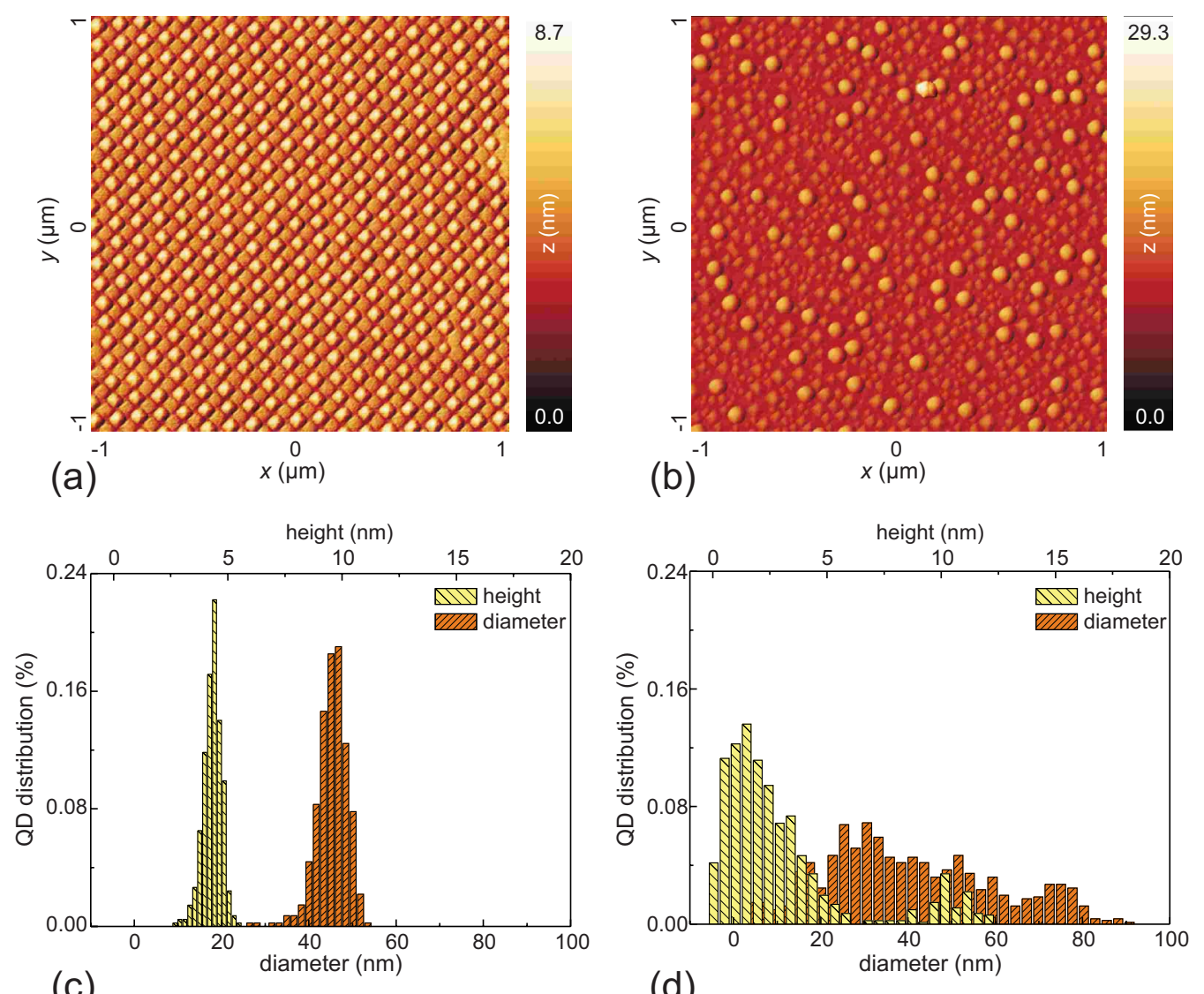

FIG. 1. (Color online) AFM micrographs of the first island layer grown in the (a) prepattern pits and in (b) an unpatterned substrate region. The resulting distributions of island height and base diameter are shown in (c) and (d), respectively.

the last island layer Ge dots deposited on a nonpatterned area of the same sample. The bimodal size distribution of dots in the first layer containing hut and dome clusters leads a strongly laterally disordered but vertically ordered dot arrangement in the course of the stacking of dot layers. The dome clusters increase in size with increasing number of island layers, even up to the size of superdomes ${ }^{51}$ at the expense of the pyramids. The AFM scan [Fig. 2(d)] shows that large dome clusters exist on the surface, with some pyramids nucleating in the surrounding, whereas most of the area in between has only a low dot density. The $10 \mathrm{~nm}$ spacer layer is not sufficiently thick in this case to yield a planar surface on top of dome clusters, thus the stacking leads to a rather wavy surface as seen in the TEM [Fig. 2(b)]. We assign the preferred nucleation of huts in the neighborhood of the dome clusters to this waviness since it leads to an enhanced number of surface steps. However, this wavy structure makes it impossible to evaluate the size distribution of these islands with reasonable statistics.

In order to obtain more detailed information on the structural perfection of the Ge quantum dot crystals, x-ray diffraction was employed at the Troika II beamline at the European Synchrotron Radiation Facility. A double crystal diamond (111) monochromator is used to select a wavelength of $1.54 \AA$, higher harmonics are suppressed by a pair of $\mathrm{Pd}$ mirrors. Two reciprocal-space maps in the [110] azimuth were recorded around the symmetrical (004) and the asym- metrical (224) Bragg peaks, using a positional sensitive detector, which is recording simultaneously the intensity along a line along the scattering angle, thus allowing to use long counting times to obtain good intensity statistics. The associated maps are shown in Figs. 3(a) and 3(b), respectively. In these figures, $Q_{x}$ and $Q_{z}$ are the coordinates of the scattering vector $\mathbf{Q}=\mathbf{K}_{f}-\mathbf{K}_{i}$ parallel and perpendicular to sample surface, respectively. $\mathbf{K}_{i, f}$ are the wave vectors of the primary and scattered beams lying in the $Q_{x} Q_{z}$ scattering plane. Due to the high dynamic range of the intensity distribution, larger than that of the detector, Al attenuators of variable thicknesses are used to attenuate the incident beam when the scan crosses intense peaks. The attenuation is electronically corrected afterwards. Nevertheless, some residual stripes along $2 \theta$ direction are visible around the most intense peaks: these are instrumental artifacts caused by the resolution function of the experimental setup. The linear detector used in the measurement exhibits "cross-talk," i.e., an enhancement of the signal in a given pixel due to a very large signal in a neighboring pixel. This cross-talk gives rise to artificial streaks crossing all sharp maxima in the measured reciprocal-space map including most of the intense satellite peaks. Since the entrance window of the linear detector was perpendicular to the diffracted beam, the angle of the streaks with the $q_{z}$ axis always equals the angle of the diffracted beam with the sample surface (exit angle) and therefore differs for different Bragg reflections. 


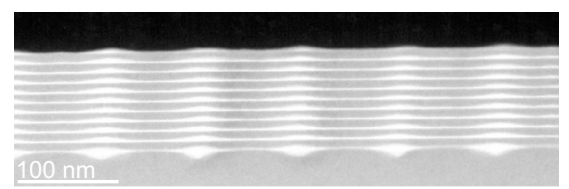

(a)

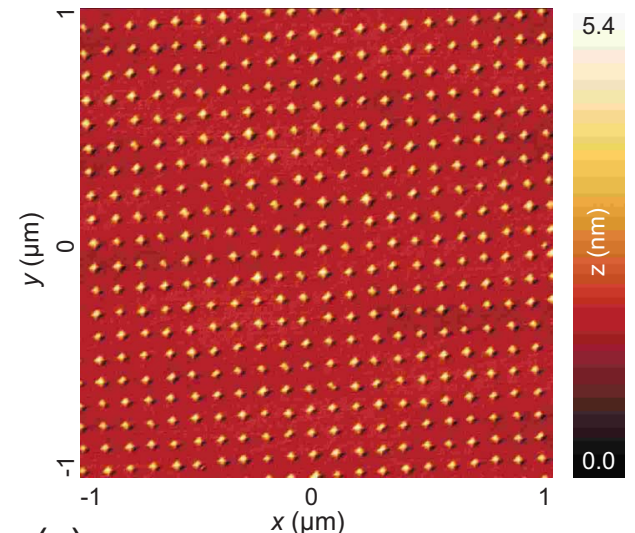

(c)

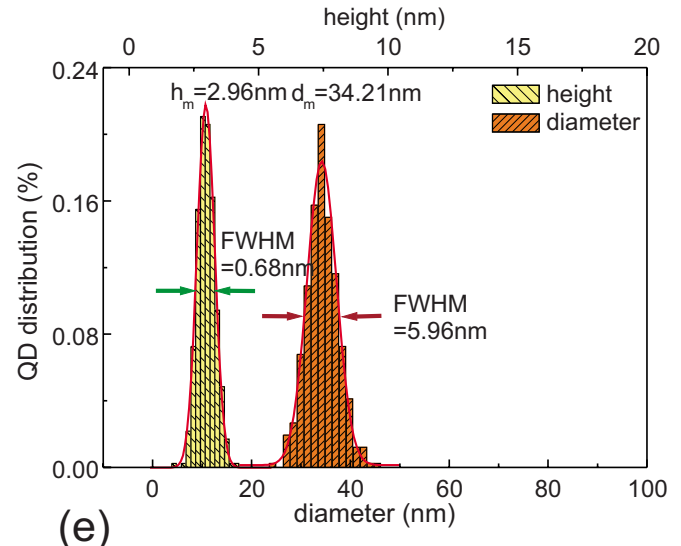

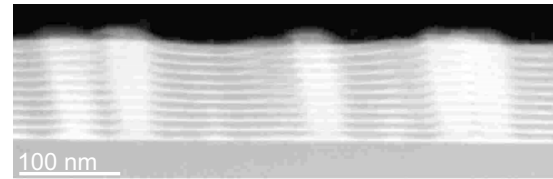

(b)

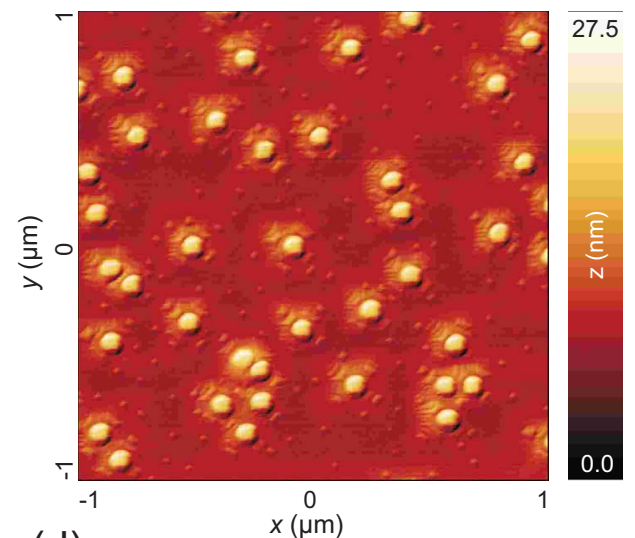

(d)

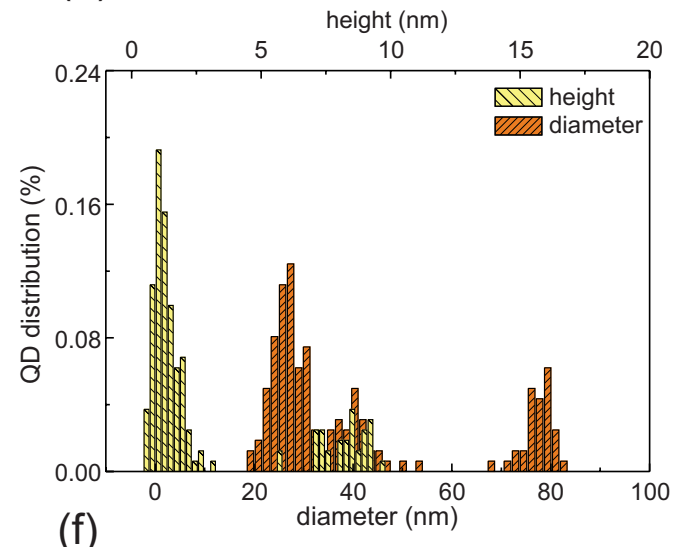

(f)

FIG. 2. (Color online) Cross-sectional transmission electron micrograph of a (a) three-dimensional dot crystal and of the (b) reference piece without prepatterning. AFM images of the topmost island layer prior to the final capping step are shown in (c) and (d) and the corresponding distributions of island height and diameter are displayed in (e) and (f).

Using an incident-beam size of $100 \times 200 \mu \mathrm{m}^{2}$, only the prepatterned area was illuminated. The regular arrangement of islands leads to a series of satellite maxima both in growth direction as well as in lateral direction. In between the vertical satellite maxima, fringes due to the total thickness of the

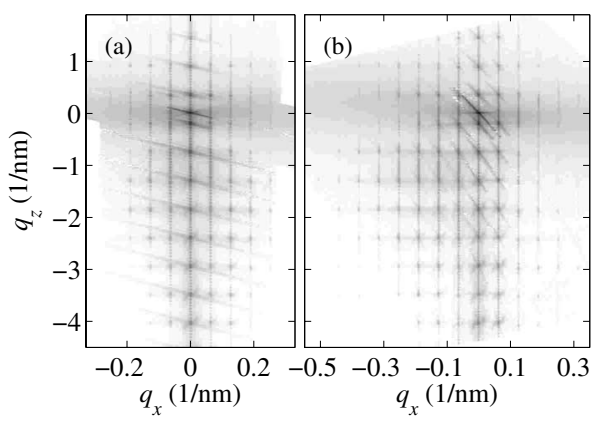

FIG. 3. Reciprocal-space maps of diffracted intensity measured in (a) (004) and (b) (224) coplanar diffractions. The oblique streaks are experimental artifacts explained in the text.
SiGe dot stack are visible even for higher lateral satellite order, indicating a high perfection of the 3D dot crystal. For the lateral and vertical periods we obtain values of $100.2 \pm 0.2$ and $11.5 \pm 0.1 \mathrm{~nm}$. Changing the azimuth by $90^{\circ}$, an in-plane period of $90.7 \mathrm{~nm}$ is found. Thus the measurements reflect rather accurately the design value of a 90 $\times 100 \mathrm{~nm}^{2}$ array. A large number of satellites resolved indicate crystalline perfection of the quantum dot crystal which is unmatched so far for $\mathrm{x}$-ray analysis of any semiconductor quantum dot system. The number of resolved satellite peaks may not be even limited by the perfection of the dot crystal but rather by the fact that the $2 \mathrm{D}$ pit pattern is not aligned perfectly with the crystalline [110] direction of the Si substrate, consequently the intensity maxima drift out of the scattering plane with increasing order, distorting the envelopes. Mapping around the (224) reciprocal-lattice point, it becomes clear that the envelope of the satellites is shifted to small $Q_{x}$ values, which is an indication of elastic relaxation in the Ge islands. An analysis of the widths of the vertical satellite peaks yields a virtually constant value identical to 
that of the substrate peak, i.e., the peak widths are limited by the instrument resolution function and not by the sample perfection, yielding a lower limit for the long-range order of dot positions of at least ten periods.

\section{STRUCTURE MODEL}

To assess the structural parameters of the 3D quantum dot crystal, we compare the measured $\mathrm{x}$-ray diffraction maps to the simulated intensity distributions. In the simulations, we have restricted ourselves to the kinematical approximation, in which multiple photon scattering and consequently x-ray refraction and absorption are neglected, and we consider that the measured signal is statistically averaged. The intensity scattered into the point $\mathbf{Q}=\mathbf{q}+\mathbf{h}$ is s $^{3,52}$

$$
I_{\mathbf{h}}(\mathbf{q})=\left\langle\left|\int_{V} d \mathbf{r}^{3} \chi_{\mathbf{h}}(\mathbf{r}) e^{-i \mathbf{h} \cdot \mathbf{u}^{\mathrm{tot}}(\mathbf{r})} e^{-i \mathbf{q} \cdot \mathbf{r}}\right|^{2}\right\rangle,
$$

where $\mathbf{u}^{\text {tot }}(\mathbf{r})$ is the total displacement field in the sample caused by all quantum dots, $\chi_{\mathbf{h}}(\mathbf{r})$ is the $\mathbf{h}$ th coefficient of the crystal polarizability, depending on the position due to the local chemical composition, and the averaging \langle\rangle is performed over all positions and shapes of the quantum dots. This expression is exact within the restrictions above, but the averaging is practically impossible to perform. Therefore, we use two approaches: (i) no statistical disorder of the dot positions and shapes, the displacement field $\mathbf{u}^{\text {tot }}$ is calculated exactly or (ii) the positions of the dots are statistically disordered; the dots are identical and in the calculation of $\mathbf{u}^{\text {tot }}$ we neglect the surface relaxation of internal stresses.

The simulation of the diffracted intensity using approach (i) was described in detail in our previous work. ${ }^{41}$ The main step in this approach is to calculate the displacement field $\mathbf{u}^{\text {tot }}(\mathbf{r})$ on the basis of assumed dot shape and local chemical composition. We have used an analytic approach for this calculation based on the solution of the elastic equilibrium equations by the Fourier method.

Now let us deal in detail with approach (ii). In this approach, the diffracted intensity is ${ }^{3,52}$

$$
I_{\mathbf{h}}(\mathbf{q}) \approx\left|F_{\mathbf{h}}(\mathbf{q})\right|^{2} G(\mathbf{q}),
$$

where

$$
\begin{aligned}
F_{\mathbf{h}}(\mathbf{q})= & \int_{V} d^{3}\left(\mathbf{r}-\mathbf{r}^{\prime}\right) e^{-i \mathbf{q} \cdot\left(\mathbf{r}-\mathbf{r}^{\prime}\right)}\left[\chi_{\mathbf{h}}\left(e^{-i \mathbf{h} \cdot \mathbf{u}\left(\mathbf{r}-\mathbf{r}^{\prime}\right)}-1\right)\right. \\
& \left.+\Delta \chi_{\mathbf{h}} \Omega\left(\mathbf{r}-\mathbf{r}^{\prime}\right) e^{-i \mathbf{h} \cdot \mathbf{u}\left(\mathbf{r}-\mathbf{r}^{\prime}\right)}\right]
\end{aligned}
$$

is the structure factor of a single-quantum dot (assumed identical for all dots in the sample) and

$$
G(\mathbf{q})=\left\langle\sum_{\mathbf{R}} \sum_{\mathbf{R}^{\prime}} e^{-i \mathbf{q} \cdot\left(\mathbf{R}-\mathbf{R}^{\prime}\right)}\right\rangle
$$

is the correlation function of the positions $\mathbf{R}$ of the quantum dots. The surface relaxation of internal stresses neglected here would modify the structure factor $F_{\mathbf{h}}(\mathbf{r})$ of the dots in the topmost layer. From this neglect it follows that the displacement $\mathbf{u}\left(\mathbf{r}-\mathbf{r}^{\prime}\right)$ of an atom in point $\mathbf{r}$ due to the dot in $\mathbf{r}^{\prime}$ depends only on $\mathbf{r}-\mathbf{r}^{\prime}$ and not on $\mathbf{r}$ and $\mathbf{r}^{\prime}$ independently. Further, in Eq. (3) we have denoted $\chi_{\mathbf{h}}$ the crystal polarizability of the host lattice, $\mathbf{h}$ is the diffraction vector (reciprocal-lattice vector), $\Omega\left(\mathbf{r}-\mathbf{r}^{\prime}\right)$ is the shape function of the dot (unity if $\mathbf{r}, \mathbf{r}^{\prime}$ belong to the same dot, zero otherwise), and $\Delta \chi_{\mathbf{h}}$ is the difference in the polarizabilities of the dot structure and the neighboring lattice, depending mainly of the Ge content in the dot.

The averaging in Eqs. (2) and (4) is performed over random-dot positions $\mathbf{R}$ and not over random-dot sizes. A statistical distribution of the dot sizes would lead to an almost constant intensity background in the simulated intensity distribution. Since this effect can hardly be separated from the background signal in the experimental data, the dot size distribution was not considered, but is instead determined from the AFM images (see Figs. 1 and 2).

Using Eqs. (2)-(4) for the diffracted intensity distribution, we have to assume a suitable structure model of a single dot, from which the structure factor can be calculated by Eq. (3), and a suitable model for the positions of the dots in the three-dimensional dot array ("dot crystal") used in the calculation of the correlation function according to Eq. (4). Using approach (ii), the structure factor follows from an assumed average dot shape and its deformation field. In the structure model of the dot positions, the lateral position $\mathbf{X}_{j_{1}, j_{2}, n}$ of the $\left(j_{1}, j_{2}\right)$ th dot at the interface $n$ is

$$
\mathbf{X}_{j_{1}, j_{2}, n}=\mathbf{X}_{j_{1}, j_{2}, n-1}+\mathbf{U}_{j_{1}, j_{2}, n}, \quad n=1, \ldots, N, \quad j_{1,2}=1, \ldots, M,
$$

where $n=0$ are the dots at the prepatterned substrate surface, $N$ is total number of the self-organized two-dimensional dot arrays, and $M^{2}$ is the number of the dots in one twodimensional dot array. The vertical coordinates of the dots are not random,

$$
Z_{j_{1}, j_{2}, n}=(n-1) D
$$

where the zero coordinate is at the substrate surface and $D$ is the (nonrandom) multilayer period.

The basic assumption of the model is that the random lateral shifts $\mathbf{U}_{j_{1}, j_{2}, n}$ of the dots are not correlated,

$$
\left\langle\mathbf{U}_{j_{1}, j_{2}, n} \cdot \mathbf{U}_{k_{1}, k_{2}, m}\right\rangle \sim \delta_{j_{1}, k_{1}} \delta_{j_{2}, k_{2}} \delta_{n, m} .
$$

Thus, the lateral position of the dot $\left(j_{1}, j_{2}, n\right)$ depends only on the position of the dot $\left(j_{1}, j_{2}, n-1\right)$ underneath (see the sketch in Fig. 4) and it is not influenced by the lateral positions of other dots $\left[\left(k_{1}, k_{2}\right) \neq\left(j_{1}, j_{2}\right), n\right]$ at the same interface. Using this simplification, we neglect the influence of the elastic strains originating both from the dots $\left[\left(k_{1}, k_{2}\right)\right.$ $\left.\neq\left(j_{1}, j_{2}\right), n\right]$ at the same interface and the dots $\left(j_{1}, j_{2}, n\right.$ $-2),\left(j_{1}, j_{2}, n-3\right), \ldots$ on the growth of the dot $\left(j_{1}, j_{2}, n\right)$.

The lateral positions of the dots $\left(j_{1}, j_{2}, 0\right)$ at the prepatterned substrate surface are induced by the prepatterning, assumed perfectly periodic. Since the dots are, however, located in the center of the pits only with a certain precision, the dot positions are not perfect and for their description a long-range-order (LRO) model is used, 


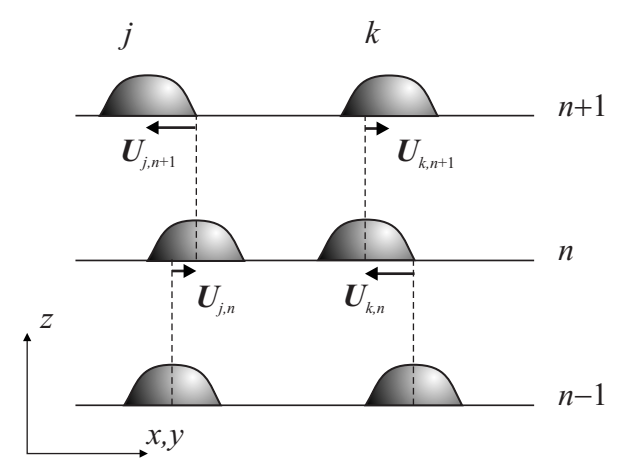

FIG. 4. Structure model of the dot positions, the $j$ th and $k$ th columns of the dots are sketched, $n-1, n$, and $n+1$ are the interface indexes.

$$
\mathbf{U}_{j_{1}, j_{2}, 0}=\mathbf{a}_{1} j_{1}+\mathbf{a}_{2} j_{2}+\mathbf{U}_{j_{1}, j_{2}, 0}
$$

where $\mathbf{a}_{1,2}$ are the basis vectors of the substrate pattern and the random displacements $\mathbf{U}_{j_{1}, j_{2}, 0}$ are not correlated,

$$
\left\langle\mathbf{U}_{j_{1}, j_{2}, 0} \cdot \mathbf{U}_{k_{1}, k_{2}, 0}\right\rangle \sim \delta_{j_{1}, j_{2}} \delta_{k_{1}, k_{2}} .
$$

Using these assumptions, we calculated the correlation function and obtained

$$
\begin{aligned}
G(\mathbf{q})= & M^{2}\left[N+1+S_{2}\left(\psi \xi, \psi^{*} / \xi\right)\right]+\left[G_{0}\left(\mathbf{q}_{\|}\right)-M^{2}\right] \\
& \times\left\{S_{1}\left(|\xi|^{2}\right)+S_{2}\left[\psi \xi,(\psi \xi)^{*}\right]\right\},
\end{aligned}
$$

where $\mathbf{q}_{\|}, q_{z}$ are the component of the vector $\mathbf{q}$ parallel and perpendicular to the sample surface, respectively,

$$
\begin{gathered}
S_{1}(a)=\sum_{n=0}^{N} a^{n}=\frac{n^{N+1}-1}{a-1}, \\
S_{2}(a, b)=2 \operatorname{Re}\left(\sum_{n=1}^{N} \sum_{m=0}^{n-1} a^{n} b^{m}\right) \\
=2 \operatorname{Re}\left(\frac{1}{b-1}\left[\frac{(a b)^{N-1}-a b}{a b-1}-\frac{a^{N+1}-a}{a-1}\right]\right),
\end{gathered}
$$

and

$$
\psi=e^{-i q_{z} D}, \quad \xi=\left\langle e^{\left.-i \mathbf{q}_{\|} \cdot \mathbf{U}_{j, n}\right\rangle_{j>0}}=\exp \left(-\sigma^{2} q_{\|}^{2} / 2\right) .\right.
$$

In the last expression, we have assumed that the displacements $\mathbf{U}_{j, n}, j>0$ are normally distributed with the rootmean-square (rms) dispersion $\sigma$, where

$$
G_{0}\left(\mathbf{q}_{\|}\right)=\left\langle\sum_{j_{1}, j_{2}} \sum_{k_{1}, k_{2}} e^{-\mathbf{i} \mathbf{q}_{\|} \cdot\left(\mathbf{U}_{j_{1}, j_{2}, 0^{-}}-\mathbf{U}_{k_{1}, k_{2}, 0}\right)}\right\rangle
$$

is the correlation function of the positions of the dots at the prepatterned substrate surface. For the calculation of this correlation function, we use a standard LRO model, yielding the expression

$$
G_{0}\left(\mathbf{q}_{\|}\right)=M^{2}\left(1-|\omega|^{2}\right)+|\omega|^{2} G_{0}^{\mathrm{id}}\left(\mathbf{q}_{\|}\right),
$$

where

$$
\omega=\left\langle e^{\left.-i \mathbf{q}_{\|} \cdot \mathbf{U}_{j_{1}, j_{2}, 0}\right\rangle}=\exp \left(-\sigma_{0}^{2} q_{\|}^{2} / 2\right)\right.
$$

and

$$
G_{0}^{\mathrm{id}}\left(\mathbf{q}_{\|}\right)=\left|\sum_{j_{1}=1}^{M} \sum_{j_{2}=1}^{M} e^{i \mathbf{q}_{\|} \cdot\left(\mathbf{a}_{1} j_{1}+\mathbf{a}_{2} j_{2}\right)}\right|^{2}
$$

is the structure factor of an ideally periodic two-dimensional dot array. This factor can be directly calculated and it exhibits maxima in the points $\mathbf{q}_{\|}$of the two-dimensional lattice reciprocal to the lattice generated by the vectors $\mathbf{a}_{1,2}$. The width of the maxima is inversely proportional to $M$. We define $M^{2}$ to be the number of the dots at each interface that are coherently irradiated by the primary wave. Then, the width of the calculated maxima of the function $G_{0}^{\text {id }}$ is affected by the reciprocal-space resolution of the experimental setup. Function $G_{0}^{\text {id }}$ calculated directly by Eq. (13) exhibits nonphysical side maxima that can be suppressed by introducing a small statistical dispersion of the value $M$ (of the order of few percent of $\langle M\rangle$ ).

The structure model described above [Eqs. (5)-(13)] is a mixture of a LRO model giving the lateral positions of the dots at the substrate surface and short-range-order (SRO) model, determining the correlation of the lateral dot positions at subsequent interfaces. In order to illustrate the properties of this model, we present in Fig. 5 the simulation of the function $G\left(q_{x}, 0, q_{z}\right)$ assuming a square pattern of the substrate surface $\mathbf{a}_{1}=(a, 0,0), \mathbf{a}_{2}=(0, a, 0), a=100 \mathrm{~nm}$; the superlattice period was $D=10 \mathrm{~nm}$. From the figure it follows that the lateral FWHMs $\Delta q_{x}$ of the lateral satellites are constant and they are determined only by the value of $M$. The rms dispersion $\sigma_{0}$ determines the profile of the function $G\left(q_{x}, 0, q_{z}=\right.$ const $)$ between the satellites. The vertical FWHMs $\Delta q_{z}$ of the lateral satellites depend on the $q_{x}$ coordinate of the satellite $q_{x}^{(p)}=2 \pi p / a$, where $p$ is the order of the satellite on the number $N$ of the periods, as well as on the superlattice period $D$. This dependence is rather complicated; for very large $N, \Delta q_{z}$ converges to the $p^{2}$ dependence $\Delta q_{z}$ $\rightarrow\left(q_{x}^{(p)}\right)^{2} \sigma^{2} / 2$ similar to the standard SRO model.

Summarizing this part, the structure model describing the ordering of the quantum dots in a three-dimensional array has the following parameters: $D$-vertical superlattice period, $a$-the period of the square dot array at each interface, $\sigma_{0}$-the rms deviation of the dot positions at the prepatterned substrate surface from the points of an ideal square array, and $\sigma$-the rms deviation from the ideal vertical replication of the dot positions at subsequent interfaces.

\section{ANALYSIS OF MEASURED DATA}

We have analyzed the intensity maps measured around the (004) and (224) reciprocal-lattice points (Fig. 3) and we obtained parameters of the ordering of the dots in the threedimensional dot crystal as well as characteristic parameters of a single dot.

In the analysis of the data, we used approach (ii) described in Sec. III. The main problem in this analysis is to discriminate the structure factor $F_{\mathbf{h}}(\mathbf{q})$ from the correlation function $G(\mathbf{q})$. Since the former factor is a much slower function of $\mathbf{q}$ than the correlation function, the widths of the maxima of $G(\mathbf{q})$ (satellite maxima) are not affected by the structure factor; this factor influences only the integral heights of the maxima. For the determination of the rms 


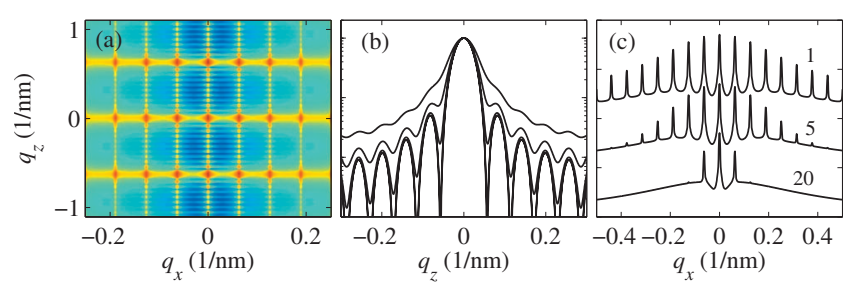

FIG. 5. (Color) (a) The two-dimensional correlation function $G\left(q_{x}, q_{z}\right)$ calculated for $\sigma=3 \mathrm{~nm}, \sigma_{0}=3 \mathrm{~nm}$, and $M=10$, (b) the vertical shape of the first four lateral satellites $(\sigma=3 \mathrm{~nm}$, normalized to the maximum value), and (c) the correlation function $G\left(q_{x}, q_{z}=0\right)$ calculated for various $\sigma_{0}$ (in nanometers).

deviation $\sigma$ we determined the dependence of the FWHMs $\Delta q_{z}$ of the lateral satellites averaged over various vertical satellites on their horizontal positions $q_{x}$ and compare this dependence with the theory above [Fig. 6(a)]. We have used both (224) and (004) intensity maps; the former map exhibits a larger number of well-pronounced satellites and it is hence better suited than (004) for the determination of $\sigma$. Moreover, the FWHMs of the (004) satellites with larger $q_{x}$ are determined with a large error due to their very low intensity. From the fit to the (224) data we obtained $\sigma=3.5 \pm 0.5 \mathrm{~nm}$.

For the determination of $\sigma_{0}$ we have used the dependence $G\left(q_{x}, 0, q_{z}=\right.$ const $)$. As shown in the theory section, the FWHMs $\Delta q_{x}$ of the lateral satellites depend only on the number $M$ of coherently irradiated dots and not on the rms deviations, which affect the intensity distributions between the satellites. However, this intensity is substantially affected also by the structure factor $F_{\mathbf{h}}(\mathbf{q})$ of a single dot. In order to eliminate this influence, from the measured data we have determined the envelope function $I_{\mathbf{h}}^{\text {env }}\left(q_{x}, q_{z}=\right.$ const $)$ of the satellites and the function $I_{\mathbf{h}}^{\text {diff }}\left(q_{x}, q_{z}=\right.$ const $)$ following the diffuse intensity between the satellites. The ratio of both functions does not contain the structure factor

$$
\frac{I_{\mathbf{h}}^{\text {env }}\left(q_{x}\right)}{I_{\mathbf{h}}^{\text {diff }}\left(q_{x}\right)}=\frac{G^{\text {env }}\left(q_{x}\right)}{G^{\text {diff }}\left(q_{x}\right)} .
$$

Thus, this ratio depends only on $\sigma_{0}$ and $M$. From the fit of the values determined both from the (224) and (004) maps to the theory we obtained $\sigma_{0}=3.0 \pm 1.0 \mathrm{~nm}$ and $M=6 \pm 1$, cor-
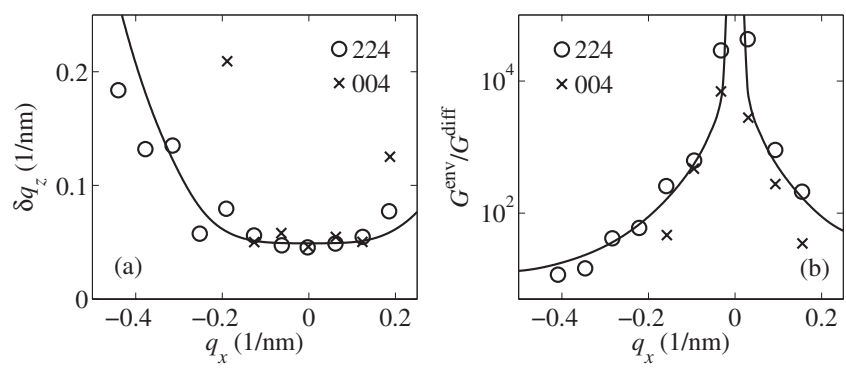

FIG. 6. (a) The dependence of the vertical FWHMs $\Delta q_{z}$ of the lateral satellites on $q_{x}$, determined from (004) and (224) reciprocalspace maps (points) and its fit by the theory (line). (b) The ratio of the intensity of the satellites and the intensity of the background between the satellites determined from the measured intensity maps (points) and its fit by the theory (line).

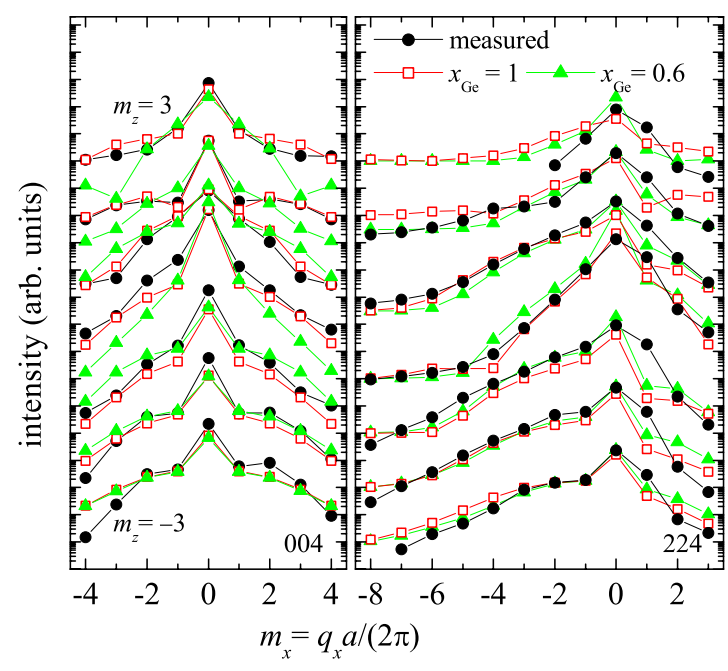

FIG. 7. (Color online) The integrated intensities of the satellites calculated for various local Ge concentrations in the dot volumes, using the dot shapes specified in the text (open squares and solid triangles), the measured intensities are denoted by black points.

responding to the size of the coherently irradiated sample surface of $0.6 \mu \mathrm{m}$ [see Fig. 6(b)].

We have used the integrated intensities of the satellites measured both in (004) and (224) diffractions for the determination of the local chemical composition of the dots. For this purpose, we have simulated the integrated intensities us-
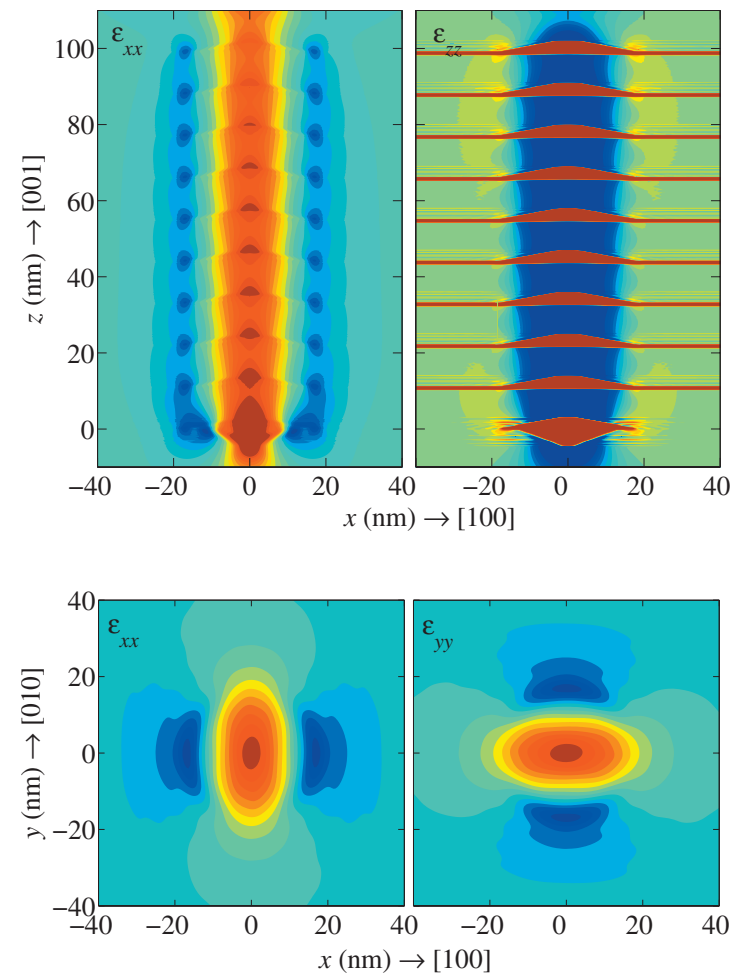

FIG. 8. (Color) Components $\varepsilon_{x x, z z}$ of the strain tensor calculated in the $x z$ plane and $\varepsilon_{x x, y y}$ in the $x y$ plane for $z=56 \mathrm{~nm}$. The contour step is $5 \times 10^{-4}$ and the positive (negative) values are denoted by red (blue) colors. The free surface is at $z=110 \mathrm{~nm}$; the different shape of the lowermost dot is clearly visible. 
ing the exact approach (i). In the simulation we assumed that the dots have shapes of truncated cones, their diameter and height have been taken from the AFM measurements (34 and $3 \mathrm{~nm}$, respectively), and the diameter of the top facet was 5 $\mathrm{nm}$. Assuming rotational symmetry instead of a quadratic one actually has no big influence on the obtained results as long as only one azimuth is concerned. ${ }^{53}$ Only if both [100] and [110] azimuths are fitted simultaneously, the difference in the aspect ratios has to be taken into account. The dots in the first layer, grown within the pits of the prepattern, have different shapes-from the TEM image in Fig. 2(a) it follows that these dots can be modeled by a pair of truncated cones; the additional cone is oriented downward, its height is $4 \mathrm{~nm}$, and its diameters at the base and at the apex are 20 and $2 \mathrm{~nm}$. Figure 7 shows the results of the simulations performed for various Ge concentrations. The best agreement of the measured and simulated integrated intensities was achieved for a mean Ge concentration in the dots of $x_{\mathrm{Ge}}=60 \%$.

Based on this chemical composition and on the dot shapes described above, we have calculated the components of the strain tensor,

$$
\varepsilon_{j k}=\frac{1}{2}\left(\frac{\partial u_{j}^{\mathrm{tot}}}{\partial x_{k}}+\frac{\partial u_{k}^{\mathrm{tot}}}{\partial x_{j}}\right), \quad j, k=x, y, z .
$$

The results are plotted in Fig. 8. From the simulations we obtained the maximum vertical and lateral strains in the dot volumes $\varepsilon_{j k \max } \approx 0.033$ and $\varepsilon_{x x \max } \approx 0.01$. In the spacer layer between the dots the maximum strain values are negative: $\varepsilon_{z z \max } \approx-0.01$ and $\varepsilon_{x x \max } \approx-0.004$. The values of the strain components are calculated with respect to the nondeformed silicon lattice.

\section{AFM MEASUREMENTS}

In order to cross-check how much results on the dot position fluctuations obtained from AFM scans differ from the $\mathrm{XRD}$ results, we have also analyzed the AFM pictures of the dots at the prepatterned substrate surface $(n=0)$ and at the free surface of the sample $(n=N)$ using the same structure model as for the analysis of the XRD data. Let us denote $h(\mathbf{x})$ the shape function of a single dot. Then the profile of the interface $n$ obtained by AFM is

$$
H_{n}(\mathbf{x})=\sum_{j_{1}, j_{2}} h\left(\mathbf{x}-\mathbf{X}_{j_{1}, j_{2}, n}\right) .
$$

The square of the absolute value of the two-dimensional Fourier transformation of this function is given by

$$
H_{n}^{\mathrm{FT}}\left(\mathbf{Q}_{\|}\right)=\int_{S} d^{2} \mathbf{x} e^{-i \mathbf{Q}_{\|} \cdot \mathbf{x}} H_{n}(\mathbf{x}) .
$$

If the scanned area $S$ is large enough, the function $\left|H_{n}^{\mathrm{FT}}\left(\mathbf{q}_{\|}\right)\right|^{2}$ can be assumed as averaged over the statistical ensemble of all dot configurations. For a single AFM image this might not be completely true since AFM scans are usually recorded with a certain resolution, in our case $512 \times 512$ pixels.

In order to achieve enough resolution to determine roughly the shape and hence the position of the island with a certain precision, the scan range cannot be made very large,

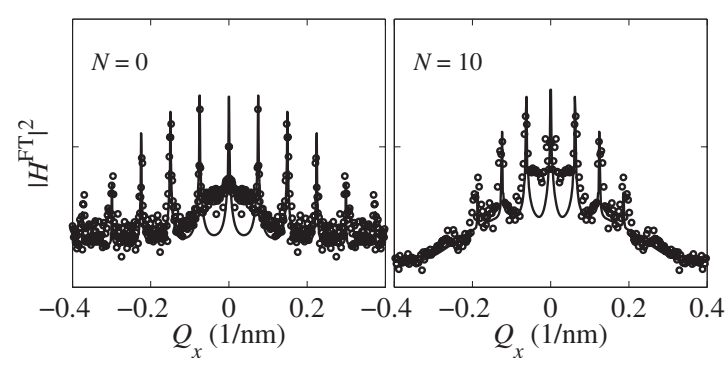

FIG. 9. The Fourier transformations of AFM pictures (points) and their theoretical simulations (lines). The AFM pictures have been taken from the first and tenth dot arrays (left and right panels, respectively).

so only a small number of islands compared to XRD experiments is measured. The formalism to analyze the position fluctuations can still be applied, but the obtained $\sigma$ values are typically smaller (i.e., the dot arrangement appears more perfect) than for analysis of large areas as in XRD.

Using the structure model described above, we obtain after some calculation

$$
\begin{aligned}
\left|H_{n}^{\mathrm{FT}}\left(\mathbf{Q}_{\|}\right)\right|^{2}= & M^{2}\left[\left\langle\left. h^{\mathrm{FT}}\left(\mathbf{Q}_{\|}\right)\right|^{2}\right\rangle-|\xi|^{2 n}\left|\left\langle h^{\mathrm{FT}}\left(\mathbf{Q}_{\|}\right)\right\rangle\right|^{2}\right] \\
& +|\xi|^{2 n}\left|\left\langle h^{\mathrm{FT}}\left(\mathbf{Q}_{\|}\right)\right\rangle\right|^{2} G_{0}\left(\mathbf{Q}_{\|}\right),
\end{aligned}
$$

where the functions $G_{0}\left(\mathbf{Q}_{\|}\right)$and $\xi$ are defined above. The last expression takes into account a statistical distribution of dot sizes that gives rise a distinct background of the function $\left|H_{n}^{\mathrm{FT}}\left(\mathbf{Q}_{\|}\right)\right|^{2}$ between the satellites.

From the AFM pictures of the dot array at the substrate surface and at the free sample surface we have calculated the Fourier transformations and from the square of this Fourier transformation we have extracted linear scans crossing the satellites. In Fig. 9 we have fitted these scans to the theory; in the fitted curves we have assumed the dot shape specified above. The scans of the substrate surface $(n=0)$ depend only on $\sigma_{0}$ (via the correlation function $G_{0}$ ). From these curves we obtained $\sigma_{0}=1 \pm 1 \mathrm{~nm}$, smaller than the value from XRD due to the limited area of the AFM scan (see above).

The function $\left|H_{n}^{\mathrm{FT}}\left(\mathbf{Q}_{\|}\right)\right|^{2}$ for $n>0$ depends both on $\sigma$ and $\sigma_{0}$ and it is impossible to determine these two parameters simultaneously. Therefore, we determined the value of $\sigma$ using the $\sigma_{0}$ values determined from the AFM data of the substrate surface. We used the same procedure for the AFM picture of the free sample surface (Fig. 2) and we obtained $\sigma=4 \pm 1 \mathrm{~nm}$, in reasonable agreement with the XRD results.

\section{DISCUSSION}

Considering the ordering of islands in prepatterned arrays, we have to distinguish several ordering directions: the lateral ordering within one layer, the vertical ordering of islands on top of each other, and the lateral ordering of islands on top of each other. In the first layer, the lateral ordering of the islands is determined by the prepattern pits and is clearly of longrange type, with an rms deviation from ideal lattice sites of $\sigma_{0}=3 \pm 1 \mathrm{~nm}$. Also the vertical ordering of islands is almost perfect since it depends on the control of the spacer layer thickness in MBE, which is excellent. However, the lateral 
alignment of islands in subsequent layers is governed by the strain fields of the buried ones, and since these are rather weak, the resulting ordering is of short-range type. This short-range ordering determines finally how well the lateral ordering of islands in the subsequent island layers can be controlled. In our case, we find $\sigma$ values of the order of 3 $\mathrm{nm}$, which means that the long-range order perfection of the first layer is rather well preserved in the subsequent layers: actually, the alignment of islands due to the strain fields of buried islands is very comparable to the alignment achieved by the prepattern in the first layer. Nevertheless the resulting ordering is of short-range type and consequently the disorder increases with the number of layers.

Overall, the results show that the ordering of the islands is very good, but not perfect. The main question in view of applications is then how this disorder affects the electronic properties. In order to achieve a "quantum dot crystal" in the electronic sense, wave functions of neighboring quantum dots must overlap in order to form minibands. This requires that the energy levels of each island are very similar. We have used the nextnano ${ }^{3}$ program package ${ }^{54}$ to calculate the energy levels of electrons and holes in the quantum dot (QD) crystal. Based on the structural data determined by the X-ray analysis, an excellent agreement between measured and calculated PL transition energy was obtained. ${ }^{44}$ In the ideal case, with all dots exactly of the same shape and composition and localized on ideal lattice sites, we have shown ${ }^{44}$ that the electronic $\Delta_{x y}$ states originating from the Si conduction-band minima in the $x$ directions perpendicular to the growth direction couple vertically between the layers and form delocalized states along a complete QD column.

Due to the disorder, two things may happen: (i) the distance between laterally neighboring islands is different, changing the strain fields and hence also the electronic properties; (ii) two vertically neighboring islands are not aligned exactly on top of each other. This may affect the electronic coupling first via the difference in the total strain fields, as in case (i), and in addition may influence the geometrical overlap of wave functions of the individual quantum dots. To investigate case (i), we have calculated the energy levels in strictly periodic dot arrangements, with periods varying from 90 to $110 \mathrm{~nm}$. The calculated energy levels are equal within the numeric precision of nextnano ${ }^{3}$, i.e., the lateral distances of the islands are so large that the coupling of the strain fields is negligible. For smaller lateral dot distances around $10 \mathrm{~nm}$, the extended states of two neighboring QD columns start to couple, however, coupling trough a QD column requires still smaller dots. To investigate case (ii), we have calculated the energy levels for a column of islands, where the central one is shifted out of its ideal position by $3 \mathrm{~nm}$, i.e., by $\sigma_{\text {rms }}$.

As a consequence, the ground-state energy (measured from the average valence band energy in $\mathrm{Si}$ ) is shifted by less than $1 \mathrm{meV}$ with respect to the ideal case. This shift is smaller than the energy spread due to size fluctuations that is estimated to be $3 \mathrm{meV}$ for the $\Delta_{x y}$ states and it does not affect the coupling of orbitals and the formation of minibands since their width can be estimated from nextnano ${ }^{3}$ simulations to be between 5 and $10 \mathrm{meV}$.

\section{CONCLUSION}

The combination of prepatterning and self-organized island nucleation "guided" by strain fields from buried ones leads to very regular $3 \mathrm{D}$ arrangements of quantum dots. The small distance between the quantum dots leads to a coupling of their electronic states, forming "quantum dot crystals" also in the electronic sense. A detailed investigation of the fluctuations of dot positions from layer to layer, and within one layer, from x-ray diffraction reciprocal-space maps reveals that the $3 \mathrm{D}$ arrangements of quantum dots is not perfect, but position fluctuations with an rms deviation of $3 \mathrm{~nm}$ from layer to layer exist, i.e., the vertical island coupling has short-range ordering character. From AFM measurements, also the dot size fluctuations are determined. The actual fluctuations are small enough not to suppress the coupling of electronic states into bands, hence the fabrication scheme is well suited to achieve quantum dot crystals with tailored electronic properties. The combination of $\mathrm{x}$-ray diffraction and AFM analysis proves an ideal tool for a complete structural characterization of quantum dot arrays.

\section{ACKNOWLEDGMENTS}

We thank the staff of beamline ID10B (ESRF Grenoble) for help with the setup. The financial support of these studies by the FWF Vienna (Contract No. SFB025), the Swiss National Science Foundation (Contract No. 200020-101778), the Ministry of Education of Czech Republic (Project No. MSM 0021620834), and the European Community (dDOTFET project) is acknowledged. The EUV-IL was performed at the Swiss Light Source (SLS) at the Paul Scherrer Institut.
${ }^{1}$ V. A. Shchukin and D. Bimberg, Rev. Mod. Phys. 71, 1125 (1999).

${ }^{2}$ C. Teichert, Phys. Rep. 365, 335 (2002).

${ }^{3}$ J. Stangl, V. Holý, and G. Bauer, Rev. Mod. Phys. 76, 725 (2004).

${ }^{4}$ G. Springholz, C. R. Phys. 6, 89 (2005).

${ }^{5}$ Q. Xie, A. Madhukar, P. Chen, and N. P. Kobayashi, Phys. Rev. Lett. 75, 2542 (1995).

${ }^{6}$ G. S. Solomon, J. A. Trezza, A. F. Marshall, and J. S. Harris, Jr.,
Phys. Rev. Lett. 76, 952 (1996).

${ }^{7}$ J. Tersoff, C. Teichert, and M. G. Lagally, Phys. Rev. Lett. 76, 1675 (1996).

${ }^{8}$ F. Liu, S. E. Davenport, H. M. Evans, and M. G. Lagally, Phys. Rev. Lett. 82, 2528 (1999).

${ }^{9}$ O. G. Schmidt, K. Eberl, and Y. Rau, Phys. Rev. B 62, 16715 (2000).

${ }^{10}$ O. G. Schmidt and K. Eberl, Phys. Rev. B 61, 13721 (2000).

${ }^{11}$ V. Holý, G. Springholz, M. Pinczolits, and G. Bauer, Phys. Rev. 
Lett. 83, 356 (1999).

${ }^{12}$ C. Priester, Phys. Rev. B 63, 153303 (2001).

${ }^{13}$ M. Meixner and E. Schöll, Phys. Rev. B 67, 121202(R) (2003).

${ }^{14}$ R. Kunert and E. Schöll, Appl. Phys. Lett. 89, 153103 (2006).

${ }^{15}$ H. Heidemeyer, U. Denker, C. Müller, and O. G. Schmidt, Phys. Rev. Lett. 91, 196103 (2003).

${ }^{16}$ C. Teichert, M. G. Lagally, L. J. Peticolas, J. C. Bean, and J. Tersoff, Phys. Rev. B 53, 16334 (1996).

${ }^{17}$ A. A. Darhuber, V. Holý, J. Stangl, G. Bauer, A. Krost, F. Heinrichsdorff, M. Grundmann, D. Bimberg, V. M. Ustinov, P. S. Kop'ev, A. O. Kosogov, and P. Werner, Appl. Phys. Lett. 70, 955 (1997).

${ }^{18}$ P. Schittenhelm, C. Engel, F. Findeis, G. Abstreiter, A. A. Darhuber, G. Bauer, A. O. Kosogov, and P. Werner, J. Vac. Sci. Technol. B 16, 1575 (1998).

${ }^{19}$ A. A. Darhuber, P. Schittenhelm, V. Holy, J. Stangl, G. Bauer, and G. Abstreiter, Phys. Rev. B 55, 15652 (1997).

${ }^{20}$ J. Stangl, T. Roch, G. Bauer, I. Kegel, T. H. Metzger, O. G. Schmidt, K. Eberl, O. Kienzle, and F. Ernst, Appl. Phys. Lett. 77, 3953 (2000).

${ }^{21}$ V. Holý, J. Stangl, Z. Zerlauth, G. Bauer, N. Darowski, D. Lübbert, and U. Pietsch, J. Phys. D 32, A234 (1999).

${ }^{22}$ X.-D. Wang, N. Liu, C. K. Shih, S. Govindaraju, and A. L. Holmes, Jr., Appl. Phys. Lett. 85, 1356 (2004).

${ }^{23}$ M. Schmidbauer, Sh. Seydmohamadi, D. Grigoriev, Zh. M. Wang, Yu. I. Mazur, P. Schäfer, M. Hanke, R. Köhler, and G. J. Salamo, Phys. Rev. Lett. 96, 066108 (2006).

${ }^{24}$ M. Strassburg, V. Kutzer, U. W. Pohl, A. Hoffmann, I. Broser, N. N. Ledentsov, D. Bimberg, A. Rosenauer, U. Fischer, D. Gerthsen, I. L. Krestnikov, M. V. Maximov, P. S. Kop'ev, and Zh. I. Alverov, Appl. Phys. Lett. 72, 942 (1998).

${ }^{25}$ I. L. Krestnikov, M. Strassburg, M. Caesar, A. Hoffmann, U. W. Pohl, D. Bimberg, N. N. Ledentsov, P. S. Kopev, Z. I. Alferov, D. Litvinov, A. Rosenauer, and D. Gerthsen, Phys. Rev. B 60, 8695 (1999).

${ }^{26}$ G. Mackowski, T. Karczewski, J. Wojtowicz, J. Kossut, S. Kret, A. Szczepanska, P. Duczewski, G. Prechtl, and W. Heiss, Appl. Phys. Lett. 78, 3884 (2001).

${ }^{27}$ G. Springholz, V. Holý, M. Pinczolits, and G. Bauer, Science 282, 734 (1998).

${ }^{28}$ P. Sutter, E. Mateeva-Sutter, and L. Vescan, Appl. Phys. Lett. 78, 1736 (2001).

${ }^{29}$ G. Springholz, M. Pinczolits, P. Mayer, V. Holý, G. Bauer, H. H. Kang, and L. Salamanca-Riba, Phys. Rev. Lett. 84, 4669 (2000).

${ }^{30}$ T. I. Kamins, R. S. Williams, and D. P. Basile, Nanotechnology 10, 117 (1999).

${ }^{31}$ H. Lee, J. A. Johnson, J. S. Speck, and P. M. Petroff, J. Vac. Sci. Technol. B 18, 2193 (2000).

${ }^{32}$ O. G. Schmidt, N. Y. Jin-Phillipp, C. Lange, U. Denker, K.
Eberl, R. Schreiner, H. Grabeldinger, and H. Schweizer, Appl. Phys. Lett. 77, 4139 (2000).

${ }^{33}$ Z. Zhong, A. Halilovic, T. Fromherz, F. Schäffler, and G. Bauer, Appl. Phys. Lett. 82, 4779 (2003); Z. Zhong and G. Bauer, ibid. 84, 1922 (2004).

${ }^{34}$ J. L. Gray, S. Atha, R. Hull, and J. A. Floro, Nano Lett. 4, 2447 (2004).

${ }^{35}$ A. Karmous, A. Cuenat, A. Ronda, I. Berbezier, S. Atha, and R. Hull, Appl. Phys. Lett. 85, 6401 (2004).

${ }^{36}$ M. H. Baier, S. Watanabe, E. Pelucchi, and E. Kapon, Appl. Phys. Lett. 84, 1943 (2004).

${ }^{37}$ Z. Zhong, W. Schwinger, F. Schäffler, G. Bauer, G. Vastola, F. Montalenti, and L. Miglio, Phys. Rev. Lett. 98, 176102 (2007).

${ }^{38}$ S. Kiravittaya, A. Rastelli, and O. G. Schmidt, Appl. Phys. Lett. 87, 243112 (2005).

${ }^{39}$ J. J. Zhang, M. Stoffel, A. Rastelli, O. G. Schmidt, V. Jovanovic, L. K. Nanver, and G. Bauer, Appl. Phys. Lett. 91, 173115 (2007).

${ }^{40}$ S. Kiravittaya, A. Rastelli, and O. G. Schmidt, Appl. Phys. Lett. 88, 043112 (2006).

${ }^{41}$ J. Novák, V. Holý, J. Stangl, T. Fromherz, Z. Zhong, G. Chen, G. Bauer, and B. Struth, J. Appl. Phys. 98, 073517 (2005).

${ }^{42}$ Z. Zhong, G. Chen, J. Stangl, T. Fromherz, F. Schäffler, and G. Bauer, Physica E 21, 588 (2004).

${ }^{43}$ C. Dais, H. H. Solak, Y. Ekinci, E. Müller, H. Sigg, and D. Grützmacher, Surf. Sci. 601, 2787 (2006).

${ }^{44}$ D. Grützmacher, T. Fromherz, C. Dais, J. Stangl, E. Muller, Y. Ekinci, H. H. Solak, H. Sigg, R. T. Lechner, E. Wintersberger, S. Birner, V. Holý, and G. Bauer, Nano Lett. 7, 3150 (2007).

${ }^{45}$ O. G. Schmidt and K. Eberl, IEEE Trans. Electron Devices 48, 1175 (2001).

${ }^{46}$ K. L. Wang, J. Nanosci. Nanotechnol. 2, 235 (2002).

${ }^{47}$ M. Friesen, P. Rugheimer, D. E. Savage, M. G. Lagally, D. W. van der Weide, R. Joynt, and M. A. Eriksson, Phys. Rev. B 67, 121301(R) (2003).

${ }^{48}$ B. E. Kane, Nature (London) 393, 133 (1998).

${ }^{49}$ H. H. Solak, C. David, J. Gobrecht, V. Golovkina, F. Cerrina, K. O. Kim, and P. F. Nealey, Microelectron. Eng. 67-68, 56 (2003).

${ }^{50}$ H. H. Solak, Microelectron. Eng. 78-79, 410 (2005).

${ }^{51}$ T. I. Kamins, G. Medeiros-Ribeiro, D. A. A. Ohlberg, and R. S. Williams, J. Appl. Phys. 85, 1159 (1999).

${ }^{52}$ U. Pietsch, V. Holý, and T. Baumbach, High Resolution X-ray Scattering from Thin Films and Nanostructures (Springer, New York, 2004).

${ }^{53}$ A. Hesse, J. Stangl, V. Holý, T. Roch, G. Bauer, O. G. Schmidt, U. Denker, and B. Struth, Phys. Rev. B 66, 085321 (2002).

${ }^{54}$ J. A. Majewski, S. Birner, A. Trellakis, M. Sabathil, and P. Vogl, Phys. Status Solidi C 1, 2003 (2004). 